\title{
Dendritic Cell Vaccines Presenting Autologous Tumor Antigens from Self-renewing Cancer Cells in Metastatic Renal Cell Carcinoma
}

\author{
Robert O. Dillman ${ }^{1,2^{*}}$ and Carol Depriest ${ }^{3}$ \\ ${ }^{1}$ Hoag Cancer Institute, Newport Beach, CA, USA; ${ }^{2}$ AIVITA Biomedical, Inc. Irvine, CA, USA; \\ ${ }^{3}$ Hoag Institute for Research and Education, Franklin, TN, USA
}

\begin{abstract}
Background and objectives: Metastatic renal cell cancer is typically resistant to chemotherapy but does respond to vascular endothelial growth factor receptor signal transduction inhibition and to a variety of immunotherapies, including interleukin (IL)-2 and monoclonal antibodies that inhibit immune checkpoints. Enhanced immune recognition of tumor antigens may improve clinical outcomes. The objective of this study was to investigate the effects of a patient-specific approach utilizing autologous dendritic cell vaccines and self-renewing autologous tumor cells in patients with metastatic renal cell carcinoma.
\end{abstract}

Methods: Short-term cell lines were established from resected renal cell cancer specimens. Autologous dendritic cells were derived from peripheral blood mononuclear cells cultured in granulocyte-macrophage colonystimulating factor (GM-CSF) and IL-4. Antigen loading was accomplished by incubating irradiated tumor cells with dendritic cells. The vaccine was admixed with GM-CSF and injected subcutaneously weekly for 3 weeks, then monthly for 5 months.

Results: Short-term cell lines were established for 28 patients. Dendritic cells were produced for 11 patients. Nine patients were referred for treatment. The nine patients received 58 injections, and 6 received all 8 planned doses. Treatments were well-tolerated, other than mild to moderate injection site reactions. One patient experienced an anaphylactoid reaction attributed to microaggregates in GM-CSF. Three patients had conversion from negative to positive for a delayed-type hypersensitivity test to intradermal injection of one million autologous irradiated tumor cells. Two of seven patients had delayed complete regression of measurable oligometastatic disease. Three patients were still alive and disease-free after 5 years.

Conclusions: This patient-specific vaccine approach is feasible, well-tolerated, and associated with encouraging long-term survival in some patients. Larger trials of dendritic cells with autologous tumor antigens derived from self-renewing tumor cells, rather than bulk tumor, may be warranted.

Keywords: Carcinoma; Renal cell; Immunotherapy; Cancer vaccines; Dendritic cells; Autoantigens

Abbreviations: AE, adverse event; DC, dendritic cell; DCV, dendritic cell vaccine; DTH, delayed type hypersensitivity; GM-CSF, granulocyte-macrophage colony-stimulating factor; i.d., intradermal; IFN, interferon; IL, interleukin; ITC, irradiated tumor cell; mRCC, metastatic renal cell cancer; mTOR, mammalian target of rapamycin; PBMC, peripheral blood mononuclear cell; PFS, progression-free survival; RECIST, Response Evaluation Criteria in Solid Tumors; SAE, severe adverse event; TCV, tumor cell vaccine; TKI, tyrosine kinase inhibitor.

Received: May 07, 2018; Revised: July 03, 2018; Accepted: July 30, 2018

*Correspondence to: Robert O. Dillman, AIVITA Biomedical Inc., 18301 Von Karman, Suite 130, Irvine, CA 92612, USA. Tel: 949-872-2555; Fax: 949-258-5679; Email: bob@aivitabiomedical.com

How to cite this article: Dillman RO, Depriest C. Dendritic Cell Vaccines Presenting Autologous Tumor Antigens from Self-renewing Cancer Cells in Metastatic Renal Cell Carcinoma. J Explor Res Pharmacol 2018;3(4):96-104. doi: 10.14218/ JERP.2018.00012.
Introduction

Prior to the new millennium, metastatic renal cell cancer (mRCC) was a highly lethal disease with a 5-year survival rate of less than $10 \% .{ }^{1}$ Chemotherapy was considered ineffective, and the standard therapies were interferon (IFN)- $\alpha$ ) and high-dose interleukin (IL)-2, but the latter was not an option for many patients because of its severe side effects. IFN- $\alpha$ produced response rates of only $5 \%$ to $10 \%$, but in a 360-patient randomized trial it was associated with a higher response rate, longer median survival ( 8.5 months $v s .6$ months), and greater 1-year survival (43\% vs. 31\%) compared to medroxyprogesterone acetate hormonal therapy. ${ }^{2} \mathrm{IL}-2$ received regulatory approval for $\mathrm{mRCC}$ in May 1992 based on a response rate of $15 \%$ among 255 patients enrolled in seven different trials, and especially the durabil- 
ity of those responses. ${ }^{3}$ Subsequent reports described response rates of $19 \%$ and $23 \%,{ }^{4,5}$ and as high as $48 \%$ in more favorable subsets. ${ }^{6}$ However, at a national level neither of these agents significantly improved survival for the population of $\mathrm{mRCC}$ patients.

Treatment of mRCC changed dramatically in 2005 after the introduction of small molecule, targeted, tyrosine kinase inhibitors (TKIs), which for several years have been the standard treatments of choice as initial and salvage therapy in $\mathrm{mRCC} .{ }^{7}$ These agents all block signal transduction pathways involving vascular endothelial growth factor, in addition to other pathways. The first of these to be approved was sorafenib, in December 2005. Currently available similar agents include sunitinib (January 2006), pazopanib (October 2009), axitnib (January 2012), and cabozantinib (April 2016). Another class of agents approved after 2005 for use in mRCC is the inhibitors of the mammalian target of rapamycin (commonly known as mTOR). These mTOR inhibitors include temsirolimus (May 2007) and everolimus (March 2009). Recently, the TKI lenvatinib (May 2016) was granted a marketing indication for use in combination with everolimus. Also approved was the anti-vascular endothelial growth factor monoclonal antibody bevacizumab (August 2009), which is marketed for use in combination with IFN- $\alpha$. In large randomized trials both sunitinib and temsirolimus were clearly superior to IFN- $\alpha .{ }^{8,9}$ None of these agents has been compared directly to high-dose IL-2, and it is doubtful there ever will be such a comparison because of the more rigorous baseline health requirements needed for IL-2 therapy.

Availability of these agents has impacted survival for mRCC patients treated in the community practice of oncology. For instance, 5-year survival rates for patients with metastatic kidney cancer at diagnosis doubled from 7.5\% $(n=40)$ during 1998-2003 to $15.8 \%(n=54)$ during $2004-2009$, median survival increased from 8 to 10 months, and survival for patients with regionally advanced disease at diagnosis increased from $56 \%(n=43)$ to $69 \%$ $(n=51) .{ }^{10}$ National data from the Surveillance Epidemiologic End Result program revealed a relative survival rate of $12 \%$ for distantdisease stage patients with cancer of the kidney and renal pelvis who were diagnosed during 2007-2013. ${ }^{11}$ These data suggest that despite the approval of so many new agents, primarily on the basis of response rates and improved progression-free survival (PFS), mRCC is still a highly lethal disease, and there is still an unmet need for agents that can improve survival further.

There is great optimism that advances in immunotherapy beyond IFN- $\alpha$ and IL- 2 will result in additional survival benefit. The monoclonal antibody nivolumab that binds to the programmed death molecule-1 immune checkpoint, was approved for the treatment of mRCC in November 2015. In an 821-patient randomized trial of nivolumab versus everolimus in patients with previouslytreated $\mathrm{mRCC}$, the programmed death molecule-1 inhibitor was associated with a higher response rate and longer overall survival. ${ }^{12}$ Recently, in a large trial of first-line therapy in mRCC, the combination of nivolumab and ipilimumab (the cytotoxic T-lymphocyte antigen-4 inhibitor) was found to be superior to sunitinib, in terms of objective response rate $(42 \% v s .27 \%)$, PFS (11.6 vs. 8.4 months), and 18 -month overall survival $(75 \%$ vs. $60 \%)$ with fewer grade 3 and 4 adverse events $(46 \%$ vs. $63 \%))^{13}$ Unfortunately, because of the toxicity anticipated for the combination of nivolumab and ipilimumab, the patients enrolled in this trial were younger and probably healthier than the general population of patients with mRCC. Furthermore, among the 550 patients treated with the combination immunotherapy, there were eight treatmentrelated deaths and $22 \%$ of patients discontinued treatment because of treatment-related toxicity. Thus, despite the improvements in survival, it is coming at the expense of significant toxicity, and even greater expense when combination therapies are used. Many practicing oncologists would prefer to treat with a programmed death molecule-1 inhibitor such as nivolumab or pembrolizumab alone rather than with a more toxic and costly combination.

It had been hoped that vaccine approaches would result in clinical benefit similar to that seen with these newer agents, but so far that has not been the case. Still, because of their lack of toxicity and different mechanism of action, there may be a niche for therapeutic vaccines, especially in combination with other therapies. ${ }^{14}$ Dendritic cells (DCs) are the most effective antigen presenting cells, and patient-specific autologous tumor cell antigens may be the best source of antigens for a vaccine therapy. ${ }^{15}$ The objective of this study was to investigate, in patients with $\mathrm{mRCC}$, the effects of a patient-specific therapeutic vaccine approach utilizing autologous DCs loaded with antigens from autologous tumor cells that had been self-renewing in a short-term cell culture prior to irradiation.

\section{Methods}

\section{Regulatory assurances}

Patients were treated according to a clinical trial that conformed to the ethical guidelines of the 1975 Declaration of Helsinki. All patients gave written informed consent prior to enrollment for treatment. The clinical protocol was approved by the Hoag Hospital Institutional Review Committee for the Protection of Human Subjects, the U.S. Food and Drug Administration (BBIND8554), and reviewers for the National Cancer Institute's Physician Data Query (clinical trial number: NCI-V01-1646). The ClinicalTrials. gov Identifier is NCt00014131.

\section{Design}

This study was designed as an open-label, single arm trial with an adaptive trial design based on evidence of delayed type hypersensitivity (DTH) reactions to intradermal (i.d.) injections of their irradiated tumor cells. The initial intent was to enroll up to 40 patients who had measurable disease at the time of enrollment, and up to 40 patients who did not have measurable disease at that time. The primary endpoints were overall survival, PFS, and objective response rates in those patients who had measurable disease per Response Evaluation Criteria in Solid Tumors (RECIST). ${ }^{16} \mathrm{Sec}-$ ondary objectives were identification of adverse events (AEs) and results of tumor DTH tests.

\section{Autologous tumor cell lines}

Surgically resected cancer specimens (kidney primary or metastatic lesion) were submitted on behalf of patients with mRCC. Tumors were transported in a prepared kit containing physiologic media supplemented with fetal bovine serum, penicillin, streptomycin, gentamicin and fungizone. After arrival in the lab, tumors were mechanically and enzymatically dissociated into single-cell suspensions and either cryopreserved or immediately put into tissue culture as previously described. ${ }^{17,18}$ Fibroblast contamination was eliminated by a combination of nutritional protein starvation and a preferential attachment procedure. ${ }^{19}$ Initial success was defined by expansion to 50 million cells. The patient's managing physician was notified once a cell line was successful, and if there was continued interest in possible treatment, the culture was expanded to 200 million cells. A portion of the cells were transferred 
into serum-free media to avoid any contamination with bovine proteins, for use in the i.d. skin tests. The remaining cells were incubated with IFN- $\gamma$ at a concentration of $1,000 \mathrm{U} / \mathrm{mL}$ for 72 hours, to increase expression of histocompatibility antigens and to induce damage associated molecules that attract DCs. ${ }^{20}$ The cells were then irradiated in a $100 \mathrm{~Gy}$ cesium irradiator. The irradiated tumor cells (ITCs) were cryopreserved for potential incubation with DCs.

\begin{abstract}
Autologous DCs
DCs were derived from peripheral blood mononuclear cells (PB$\mathrm{MCs}$ ) obtained during a single leukapheresis procedure processing 1.5 to $2 \mathrm{~L}$ blood volume. Cellular components in the PBMC product were estimated using a Coulter counter. Monocytes were enriched from the apheresis PBMC product using Ficoll density gradient centrifugation. Monocytes were differentiated into DCs over 6 days by culturing in the presence of granulocyte-macrophage colony-stimulating factor (GM-CSF) and IL-4 as previously described. ${ }^{17,18,20}$ Flow cytometry to measure fluorescence from monoclonal antibody binding to surface antigens was used to measure certain cell markers. ${ }^{20,21}$
\end{abstract}

\section{Autologous dendritic cell vaccine (DCV)}

As previously described for melanoma patients, ${ }^{20}$ a range from 150 to 350 million DCs were incubated overnight with 100 million ITCs for phagocytosis and antigen loading. Ten aliquots were prepared, with each DCV dose containing 3 to 33 million cells. Quality control sterility and endotoxin tests were performed on the final DCV product. For each patient, 10 individual DCV doses were cryopreserved in liquid nitrogen.

\section{Patient eligibility}

Key eligibility criteria for treatment were: (1) histologic diagnosis of RCC; (2) availability of a short-term autologous tumor cell line; (3) availability of monocytes derived from the patient's PBMCs; (4) referral by the managing physician for vaccine therapy; (5) willingness to travel to Newport Beach, CA, USA for DCV injections; (6) Karnofsky performance status $\geq 70$, Eastern Cooperative Oncology Group performance level of 0-2; and, (7) satisfying usual phase II clinical trial criteria for hematologic, metabolic, renal and hepatic chemistry tests. At the time of treatment, patients had to be 16 years of age or greater. Patients with brain metastases were eligible if known tumors had been controlled with therapy and they were not taking pharmacologic doses of corticosteroids. Treatment was deferred until at least 1 month after any immunosuppressive therapy, including high-dose corticosteroids, chemotherapy, or radiation therapy. Patients were tested for reactivity to common recall antigens (Candida and trichophyton), but anergy was not an exclusion criterion. Presence of measurable disease at the time of treatment was not an eligibility requirement, but patients were stratified for analysis based on whether they had measurable disease per RECIST.

\section{Treatment schedule}

At the time of each treatment, a cryopreserved vial of DCV was thawed and suspended in 500 micrograms of GM-CSF and injected within 5 hours of thawing. As in related trials, ${ }^{20,22}$ subcutaneous injections were administered during weeks 1, 2, 3, 8, 12, 16, 20 and
24. Concurrent anticancer therapy was not allowed.

\section{Follow up information}

After completion of vaccine injections, patients were followed-up in person and/or by telecommunication every 3 months to collect information regarding administration of additional anticancer therapies, any delayed severe adverse events (SAEs) that might be attributable to vaccine treatment, approximate date of disease progression, or date and cause of death if deceased. Follow up was continued for up to 5 years from the date of enrollment for surviving patients.

\section{Survival}

Tumor responses were defined according to RECIST for patients with objectively measurable disease. Patients without measurable disease were monitored for disease progression at a maximum of 3-month intervals by physical examination and photon emission tomography or computerized tomography scans and, if symptomatic, whole-body bone scan and brain magnetic resonance imaging. Managing physicians and clinical trial staff identified the first site of disease progression and a date of disease progression to estimate PFS. Overall survival was calculated from the date of baseline DTH test (1 week before the first DCV injection) to the date of death.

\section{DTH to autologous tumor cells}

At baseline, and following completion of the first three weekly vaccinations, patients underwent i.d. tests for DTH to one million ITCs that had not been exposed to IFN- $\gamma$ and had been maintained and cryopreserved in serum-free medium. Within 48 to 72 hours of injection, the test was interpreted by nursing staff as negative (no induration), weakly positive (5 to $9 \mathrm{~mm}$ induration), or positive ( $\geq 1 \mathrm{~cm}$ induration). The protocol was amended to add a third tumor DTH test after 5 months of therapy, just prior to the last vaccine injection for patients who received all eight injections.

\section{Safety}

AEs were assessed at each visit for a vaccine injection, and 4 weeks after the last injection. AEs and SAEs were classified and graded 0 to 5 per National Cancer Institute Common Toxicity Criteria for Adverse Events, version 3.0.

\section{Statistics}

Overall survival and PFS were estimated using the methods of Kaplan-Meier. The 95\% confidence intervals were determined for proportions. Means were compared using the Student's $t$-test. Proportions were compared using Fisher's exact test.

Results

\section{Tumor cell lines}

From January 2000 to December 2006, short-term tumor cell lines 
Table 1. Percentage of cells positive for HLA1 or HLA-2 and the MIF per flow cytometry, before and after incubation with IFN- $\gamma$

\begin{tabular}{lll}
\hline $\boldsymbol{n}=\mathbf{2 1}$ & Pre IFN- $\boldsymbol{\varphi}$ & Post IFN- $\boldsymbol{\varphi}$ \\
\hline HLA-1 \% & $97 \pm 7$ & $99 \pm 1$ \\
HLA-1 MIF & $359 \pm 282$ & $833 \pm 403$ \\
HLA-2 \% & $10 \pm 15$ & $84 \pm 24$ \\
HLA-2 MIF & $75 \pm 118$ & $168 \pm 153$ \\
\hline
\end{tabular}

Abbreviations: HLA, human lymphocyte antigen; IFN- $\gamma$, interferon-gamma; MIF, median intensity of fluorescence.

were established for 28 of 119 samples submitted to the laboratory. More specifically, these were characterized as 28 successes, 54 failures to grow and 9 poor quality samples, and 28 samples were discontinued. Efforts to establish cell lines were discontinued because of ineligibility for the clinical trial $(n=17)$, due to physician decision $(n=5)$, death of patients $(n=3)$, patient request $(n=2)$, and technical processing error $(n=1)$. Thus, the estimated success rate was $28 / 72$ ( $38.8 \%, 95 \% \mathrm{CI}: 27.6 \%$ to $50.2 \%)$.

The time to cell line success took 0.5 to 10.3 months, with an average of $4.0 \pm 2.3$ months to reach 50 million cells. The success rates were similar whether the cultures were started immediately after processing, or following cryopreservation: $32.8 \%$ $(19 / 58)$ for fresh samples, and 37.5\% (9/24) success rate from cryopreserved samples. The length of time to a successful culture was faster for fresh tumors, with a mean of $3.5 \pm 2.35$ months compared to $5.2 \pm 1.60$ months from cryopreserved samples $(p$ $=0.032$ ). It took an average of $5.76 \pm 2.69$ months to prepare a cell line and complete quality control testing of cells for potential incubation with DCs.

Twenty-one cell lines were incubated with 1,000 units $/ \mathrm{mL}$ of IFN- $\gamma$ for 72 hours. As can be seen in Table 1, incubation with IFN- $\gamma$ resulted in an 8-fold increase in the percentage of cells considered positive for HLA-2 expression, and an increase in median intensity of fluorescence for both HLA-1 and HLA-2.

DCs

DCs were successfully generated for $11 / 11$ patients who underwent leukapheresis to collect PBMCs. All 11 were incubated with ITCs to prepare the DCV containing DCs loaded with autologous tumor antigens. Table 2 summarizes the data for 9 patients who were subsequently treated with DCV and for two DCV products that were prepared but never administered because the patients were not referred for treatment. The apheresis PBMC products averaged $70 \%$ lymphocytes, $19 \%$ monocytes, and $11 \%$ granulocytes. The apparent decrease in viability in the final product was due to the presence of residual ITCs that were not phagocytized. Table 3 summarizes the phenotypes after incubation of DCs with ITCs. The decrease in CD11c positivity was due to dilution by residual nonphagocytosed ITCs, which did not express CD11c. The increase in median intensity of fluorescence for CD83 and CD80 was consistent with some DC maturation after incubation with ITCs.

\section{DCV therapeutic product}

DCV consisted of autologous DCs and residual ITCs that were not phagocytosed during overnight coincubation. Frozen aliquots of DCV were cryopreserved in liquid nitrogen. At the time of treatment one aliquot was thawed, and viability and cell count were determined. The cells were then washed and suspended in GMCSF for injection. Table 4 shows the averages and ranges for the first three injections for all 9 patients and for the 6 patients who received an eighth dose. There was little variation in number and viability of cells among doses for each individual patient, but there was substantial variability between patients in numbers of cells injected. The $20 \%$ to $25 \%$ nonviable cells was the result of residual ITCs that had not been phagocytosed. It took a median of 5.0 months and a mean of $4.75 \pm 3.36$ months (range: 2.00 to 9.25 months) from the time of initiation of cell culture to prepare the patient-specific vaccine product, including quality control testing on the DCV product.

\section{Clinical trial}

The first patient enrolled in October 2001 and the final patient in March 2006. One patient started treatment in 2001, three in 2002, two in 2003, one in 2004, one in 2005, and one in 2006 . Enrollment was discontinued in 2007 because of poor accrual. Accrual likely was negatively impacted by the increasing availability and efficacy of small molecule TKIs, such as sorafenib and sunitinib. The study was closed to follow up in 2009, when the last of the long-term surviving patients had been followed for 5 years.

\section{Patient characteristics}

The clinical characteristics of the 9 patients enrolled for treatment are summarized in Table 5. There were 8 men and 1 woman, with a range in ages from 29 to 74 years. All had distant mRCC. Five

Table 2. Relationship of apheresis PBMCs to DCs before and after incubation with autologous ITCs for the final treatment product

\begin{tabular}{lll}
\hline & Used for treatment, $\boldsymbol{n = 9}$ & Not used for treatment, $\boldsymbol{n}=\mathbf{2}$ \\
\hline PBMCs from apheresis & $3.9 \pm 1.1 \times 10^{9}$ & $10.2 \pm 6.9 \times 10^{9}$ \\
Lymphocytes & $70.4 \pm 6.2 \%$ & $57.9 \pm 1.6 \%$ \\
Monocytes & $19.4 \pm 4.2 \%$ & $24.5 \pm 0.6 \%$ \\
DCs pre-ITCs antigen loading & $2.1 \pm 0.6 \times 10^{8}$ & $5.0 \pm 2.8 \times 10^{8}$ \\
Viability of DC pre-ITC antigen loading & $92.6 \pm 5.7 \%$ & $99 \pm 0.0 \%$ \\
DCs post-ITCs antigen loading & $2.2 \pm 1.0 \times 10^{8}$ & $5.5 \pm 4.2 \times 10^{8}$ \\
Viability of DCs post-ITCs antigen loading & $85.0 \pm 4.8 \%$ & $96.0 \pm 5.7 \%$ \\
\hline
\end{tabular}

Abbreviations: DC, dendritic cell; ITC, irradiated tumor cells; PBMC, peripheral blood monocyte. 
Table 3. Phenotypic characterization of DCs by positivity and MIF pre and post incubation with ITCs

\begin{tabular}{|c|c|c|c|c|}
\hline & \multicolumn{2}{|c|}{ Treated patients, $N=9$} & \multicolumn{2}{|c|}{ Untreated patients, $N=2$} \\
\hline & Pre-ITC incubation, $N=6 *$ & Post-ITC incubation, $N=8^{*}$ & Pre-ITC incubation, $N=\mathbf{2}$ & Post-ITC incubation, $N=2$ \\
\hline HLAII \% & 100 & 100 & 100 & 100 \\
\hline CD80 \% & $36 \pm 21$ & $29 \pm 17$ & $42 \pm 23$ & $38 \pm 32$ \\
\hline CD80 MIF & $95 \pm 19$ & $162 \pm 55$ & $87 \pm 40$ & $130 \pm 100$ \\
\hline CD11c \% & $100 \pm 0.5$ & $86 \pm 16$ & $100 \pm 0$ & $95 \pm 5$ \\
\hline CD11c MIF & $4,061 \pm 1,893$ & $4,590 \pm 2,443$ & $6,745 \pm 680$ & $4,380 \pm 3,080$ \\
\hline CD83 \% & $7 \pm 5$ & $13 \pm 18$ & $7 \pm 0$ & $5 \pm 4$ \\
\hline CD83 MIF & $93 \pm 28$ & $190 \pm 105$ & $56 \pm 1.4$ & $117 \pm 33$ \\
\hline CD86 \% & $86 \pm 15$ & $70 \pm 20$ & $56 \pm 11$ & $55 \pm 22$ \\
\hline CD86 MIF & $2,660 \pm 1,186$ & $2,986 \pm 1,113$ & $2,098 \pm 275$ & $1,576 \pm 1,064$ \\
\hline
\end{tabular}

Abbreviations: DC, dendritic cell; ITC, irradiated tumor cell; MIF, median intensity of fluorescence. *Two of the treated patients did not have phenotype testing pre-ITC loading, and one treated patient did not have phenotype testing post-ITC loading.

Table 4. Numbers and viability of cells in dendritic cell vaccine products

\begin{tabular}{llll}
\hline & Average \# cells $\times \mathbf{1 0}^{\mathbf{6}}$ & Range of cell \# $\times \mathbf{1 0}^{\mathbf{6}}$ & \multicolumn{1}{c}{ Average viability } \\
\hline Dose \#1, $n=9$ & 15.0 & 4.5 to 31.2 & $76 \%$ \\
Dose \#2, $n=9$ & 15.6 & 5.0 to 26.4 & $78 \%$ \\
Dose \#3, $n=9$ & 15.1 & 5.0 to 27.0 & $78 \%$ \\
Dose \#8, $n=6$ & 12.9 & 3.7 to 23.0 & $77 \%$ \\
\hline
\end{tabular}

of the nine had metastatic disease at the time of initial diagnosis; the other four had recurred with $\mathrm{mRCC}$ after an earlier diagnosis. Three had previously had no treatment other than surgery.

\section{Treatment and outcomes}

Treatment and outcomes are summarized in Table 6. Initial DTH and anergy tests were performed between 16 October 2001 and 17 March 2006. These 9 patients received 58 injections, with 6 patients completing all eight planned injections. Three patients experienced conversion of their DTH tests from negative to positive; all three survived beyond 2 years, but only 1 of the 3 patients who survived 5 years had conversion of their DTH test.

\section{Patient summaries}

Patient \#1-RR had spinal compression from metastases with leg weakness at the time of initial diagnosis. He discontinued treatment after three vaccinations because of recurrent problems in the area of prior spine surgery, which necessitated another operation.

Patient \#2-HS had lung and bone lesions at the start of treatment that did not progress during treatment but did progress about 11 months after completing the series of injections. He died from progressive disease 11 months later.

Table 5. Patient characteristics at baseline

\begin{tabular}{llllll}
\hline Patient ID & Age in years & Sex & ECOG & Prior Rx in addition to nephrectomy & Measurable disease \\
\hline 1-RR & 57 & M & 2 & RT, IFN- $\alpha$ & Adrenal, pelvis, bone, brain \\
2-HS & 72 & M & 0 & None & None \\
3-DD & 67 & M & 1 & RT, IL-2, CE & Lung, Bone \\
4-CG & 53 & F & 1 & None & Neck nodes \\
5-RK & 49 & $M$ & 0 & ALT & Abdominal nodes \\
6-DC & 62 & $M$ & 1 & IL-2 & None \\
7-JO & 74 & $M$ & 0 & None & Lung \\
8-JM & 29 & $M$ & 1 & RT & Lung, abdomen \\
9-LL & 65 & $M$ & 2 & IFN $\alpha+$ Bev, Sorafenib & Lung, bone \\
\hline
\end{tabular}

Abbreviations: ALT, autologous lymphocyte therapy; Bev, bevacizumab; CE, chemoembolization; ECOG, Eastern Cooperative Oncology Group; IFN- $\alpha$, interferon-alpha; IL-2, interleukin-2; RT, radiation therapy. 
Table 6. Summary of treatment and clinical results for patients treated with autologous DCV

\begin{tabular}{lllllllll}
\hline Patient ID & \# DCV & Ave \# cells per dose $\times \mathbf{1 0}^{\mathbf{6}}$ & Baseline DTH & Post DCV DTH & Obj Resp & First PD site & PFS in months & OS in months \\
\hline 1-RR & 3 & 15.5 & Neg & Neg & PD & Bone & 1.4 & 25.5 \\
2-HS & 8 & 28.2 & Neg & Pos & - & Lung, bone & 16.4 & 27.4 \\
3-DD & 4 & 21.9 & Neg & Neg & PD & Lung, bone & 1.9 & 13.1 \\
4-CG & 8 & 11.5 & Neg & Neg & PD & Neck, RP & 1.7 & 10.4 \\
5-RK & 8 & 12.8 & Neg & Neg & SD* & Brain & 10.9 & $>60$ \\
6-DC & 8 & 4.8 & Neg & Neg & - & None & $>60$ & $>60$ \\
7-JO & 8 & 20.5 & Neg & Pos & SD* & Brain & 22.4 & $>60$ \\
8-JM & 3 & 12.4 & Neg & Neg & PD & Abdomen & 1.5 & 5.7 \\
9-LL & 8 & 8.0 & Neg & Pos & PD & Lung & 1.9 & 26.1 \\
\hline
\end{tabular}

Abbreviations: AE, adverse event; DTH, delayed type hypersensitivity test to autologous tumor cells; neg, negative; Obj Resp, objective response; OS, overall survival; PD, progressive disease; PFS, progression-free survival; Pos, positive; RP, retroperitoneum; SD, stable disease. *Subsequent delayed complete response.

Patient \#3-DD had presented with spinal compression from metastases at the time of initial diagnosis. He went off-study after four injections because of progressive disease in the bone and lung, and died about 11 months later.

Patient \#4-CG had presented with left cervical adenopathy that persisted at the start of DCV. Scans showed progression of cervical and retroperitoneal lymph nodes during DCV therapy but she completed all eight doses. She subsequently was treated with IL-2 but discontinued because of toxicity. She died 15 months from the original diagnosis, and 5 months after her last DCV injection.

Patient \#5-RK had recurred 4 years after original nephrectomy and adjuvant auto-lymphocyte therapy. ${ }^{23}$ At baseline, he had measurable pulmonary lymph node metastases that remained stable throughout treatment. These eventually resolved several months after completing the series of DCV injections. He subsequently progressed in the brain and was treated with gamma knife. After that, he remained disease free and was still alive at 5 years.

Patient \#6-DC developed bilateral lung metastases 4 years after the original diagnosis. These responded to IL-2, and a residual lung lesion was resected and was the source of ITCs for the vaccine. He had no measurable disease at the time of treatment. He remained disease free after 5 years.

Patient \#7-JO had metastatic disease that included several lung nodules (largest $3.1 \mathrm{~cm}$ in the right basilar area), and cytologicallyproven malignant pleural effusion. Following nephrectomy for a $3.5 \mathrm{~cm}$ tumor primary tumor on $06 / 09 / 03$, a computed tomography scan on $07 / 30 / 03$ showed pleural effusion and several lung nodules (largest $3.0 \mathrm{~cm}$ in the right basilar area and others measured as 2.6 $\mathrm{cm}, 2.2 \mathrm{~cm}$, and $1.7 \mathrm{~cm}$. On 12/01/03 these were measured as 2.6, 1.7 , and $1.4 \mathrm{~cm}$, and the pleural effusion had resolved. When he started the vaccine in May 2004, a $1.0 \mathrm{~cm}$ lung lesion persisted as the only site of measurable disease. This lesion remained stable initially then slowly resolved such that 7 months after starting therapy, in November 2004, his scans were interpreted as showing no metastatic disease and, therefore, in complete remission. He subsequently was diagnosed with a brain metastasis that was treated with gamma knife. He remained disease free and was still alive at 5 years.

Patient \# 8-JM had familial RCC. More than 2 years after undergoing nephrectomy, he presented with pulmonary symptoms and was found to have multiple lung metastases and recurrence in the renal bed that were present when DCV was initiated. DCV was discontinued after three doses because of progression. He subsequently was treated with high-dose IL-2. He died 2.5 years from the original diagnosis, 9 months after the diagnosis of recurrent and metastatic disease, and within 6 months of starting DCV.

Patient \#9-LL had extensive lung metastases when originally diagnosed. He had some response to sorafenib prior to starting DCV. He progressed during DCV treatment but completed all 8 doses. He subsequently again briefly responded to sunitinib; after progression, a trial of inhaled GM-CSF was discontinued because of toxicity. He survived an additional 8 months without therapy.

\section{Cutaneous tests for immunological response}

\section{DTH testing}

Two of the nine patients (\#2-HS, \#8-JM) were anergic at baseline based on skin tests for Candida and trichophyton, and none of the nine reacted to the autologous tumor DTH test at baseline. Two patients converted to a positive $(>10 \mathrm{~mm})$ test at 4 weeks (\#2-HS, \#9-LL), and another patient converted at the completion of 6 months of therapy (\#7-JO). The third patient (\#3-DD) had an intermediate skin test result at week 4 . Thus, $4 / 9$ (44.\%, 95\% CI: $11.9 \%$ to $76.9 \%$ ) showed some immune response to autologous tumor that was not present at study entry. As shown in Table 6, among the 3 patients whose tumor DTH test converted from negative to positive, all survived more than 2 years. However, of the 35 -year survivors, only 1 had conversion of the tumor DTH test.

\section{Toxicity}

The vaccine was well-tolerated except for mild to moderate pain and erythema at the injection site in $5 / 9$ patients ( 3 grade- 2,2 grade-1), and 1 patient (\#5-RK) experienced an anaphylactoid reaction. The highest grade of toxicity experienced by any individual patient was 3 with grade 0,1 with grade 1,4 with grade 2,1 with grade 3 , and 0 with grade 4 . One grade $2 \mathrm{AE}$ was bone discomfort that was attributed to GM-CSF since the patient had no bone metastases.

\#5-RK experienced a grade 3 anaphylactoid reaction after his eighth and final injection on $06 / 24 / 03$. This was reported as an SAE because management included hospitalization. He had experienced mild hives and pruritus on the neck and palms of hands during the prior week, which was responding to diphenhydramine. After the final vaccination, he left the facility in stable condition, 
but called clinical trial personnel 15-20 minutes later and reported increased rash, shortness of breath, and numbness of lips and feet. On his arrival back in the Clinic, he reported continuing numbness of lips and feet, as well as chest tightness. He was treated with oxygen and intravenous medications and then admitted to the hospital. Specific medications used in treatment were dexamethasone, diphenhydramine, cimetidine, ranitidine, and insulin. The patient responded and by the following morning had returned to baseline with mild residual rash; so, he was discharged home where he had no further sequelae. It was eventually determined this was likely due to microaggregates in the GM-CSF preparation related to manufacturing changes after the product had been transferred from Immunex to Amgen to Berlex.

\section{Discussion}

The original objectives of this study included manufacturing feasibility, immune response based on tumor DTH skin tests, safety observations, and anticancer efficacy including response rate, PFS, and overall survival. The results of this abbreviated clinical trial are being reported because it is evident that despite advances in targeted therapies, including oral TKIs targeting signal transduction pathways, mTOR inhibitors, and monoclonal antibodies to immune checkpoints, there is still an unmet need for nontoxic complementary therapies for patients with $\mathrm{mRCC}$. This report on patient-specific therapeutic DCV demonstrates feasibility, limited toxicity, and data consistent with anticancer effect in several patients with mRCC.

With regard to feasibility, the first requirement is to have a short-term cell culture as a source of antigen from self-renewing autologous tumor cells. However, the success rate in this era was only $39 \%$. This is well below the $71 \%(56 / 79)$ success rate previously reported from this laboratory. ${ }^{22}$ One reason for this may have been more rigorous criteria for determining that a cell line was malignant renal cancer cells. The laboratory had previously recognized that some putative renal cell cancer cell lines generated from primary kidney masses in the past consisted of normal renal cells rather than malignant renal cells, based on differences in the ability to tolerate freezing and thawing. Reliable, rapid establishment of cell lines would be important for potential commercialization of this approach. So, while this trial confirmed feasibility, it did not establish the reliability or practicality of the approach, at least with the cell culture strategies in use at the time of this trial. On the other hand, satisfactory apheresis PMBC products were collected for each patient and DCs were produced for all patients. The final product was intended to be all DCs, but in fact contained sizeable numbers of non-phagocytosed ITCs. It may be desirable to have a purer DC product.

Toxicity does not appear to be an issue for this or any other DCVs reported to date. In this trial the DCV was well-tolerated, other than the one anaphylactoid reaction which was attributed to the adjuvant GM-CSF. As in most studies of DCVs, the most common AEs were reactions at the injection site and various symptoms that can be characterized as flu-like, including fever, malaise, myalgias, arthralgias, bone discomfort and nausea. This constellation of symptoms has been reported regardless of the antigens being presented. ${ }^{24-26}$ Bone discomfort is a well-known side effect of GMCSF that is caused by expansion of granulocytes and monocytes in the bone marrow, and is probably not related to the DCV itself.

There was some suggestion of efficacy in this study. One patient, who was already experiencing a spontaneous regression of metastatic disease, had a delayed complete regression of the only measurable lesion that persisted. Another patient had delayed regression of pulmonary adenopathy. Neither of these occurred in a time frame to be classified as objective responses per RECIST. Similar to what has been seen in melanoma patients treated with such patient-specific DCV, there appeared to be no improvement in PFS but an apparent impact on OS. This is consistent with the hypothesis that tumor-initiating cells are being preferentially targeted with this approach, rather than antigens expressed on the more numerous terminally differentiated tumor cells that predominate in any measurable tumor lesion. As a consequence, even when such a vaccine is working, there likely will still be apparent disease progression before there might be stabilization and then regression. ${ }^{15}$ The $33 \%$ actual 5 -year survival rate is of note, in as much as the national figure was less than $10 \%$ for patients with mRCC during the years this trial was conducted. ${ }^{1}$

We previously reported results for 25 renal cell cancer patients treated with ITCs as a tumor cell vaccine (TCV) that was administered with a variety of different adjuvants, primarily GM-CSF and IFN- $\gamma .{ }^{27}$ However, 8 of those patients had only a large primary lesion or locally advanced disease that had been resected. In that study, the 14 patients with measurable disease had a median survival of 7.5 months, and only 2 (14\%) survived beyond 3 years. In contrast, in the trial reported here, among the 7 patients with measurable disease, median survival was 25.5 months, and 2 (29\%) patients survived beyond 5 years. Although the numbers are small, this is consistent with other clinical data that suggests the DCV approach is superior to the TCV approach. The latter depends on in vivo rather than ex vivo antigen loading of DCs. These results are consistent with those seen in a randomized trial that compared DCV to TCV in patients with metastatic melanoma, in which the median survival was twice as long for patients treated with DCV as opposed to TCV (43.4 vs. 20.5 months), with a $70 \%$ reduction in the risk of death. ${ }^{28}$

Others have conducted small studies of autologous DCVs in mRCC, as summarized elsewhere. ${ }^{24,25,29,30}$ Antigen sources have included shared peptides, ${ }^{31}$ allogeneic cell lines, ${ }^{32}$ autologous tumor cells, ${ }^{33}$ autologous tumor lysate, ${ }^{34}$ and autologous tumor RNA. ${ }^{35}$ The treatment used in this report differs in the use of autologous tumor cells that are self-renewing ex vivo, which may preferentially present antigens associated with tumor stem cells and early progenitor cells and/or rapidly proliferating cells. One review article identified 197 patients treated with DCVs in 14 phase I/II trials, but included patients treated with allogeneic DCs. ${ }^{24}$ A more recent review that limited analysis to those reports that included at least 6 patients and only autologous DCs came up with 12 trials involving 186 patients. ${ }^{25}$ Similar to this report, most of the trials involved small numbers of patients with substantial variability in extent of metastatic disease. Objective response rates were estimated at $6 \%$ to $13 \%$ in the two meta-analyses. Most AEs were local injection site reactions and flu-like symptoms; less common were myalgias, fatigue, bone or articular pains. Few toxicities were detected above grade 2 .

The largest experience with a DCV for $\mathrm{mRCC}$ has not been published but information is available from press releases. The product is a DCV with the generic name rocapuldencel-T, previously referred to as AGS-003 (Argos Therapeutics). Autologous DCs are transfected with amplified mRNA from autologous tumors and RNA for a synthetic truncated human CD40 ligand. This DCV is injected i.d. The phase III randomized trial (known as 'ADAPT') tested the DCV as front-line treatment in patients with mRCC. The trial enrolled 462 patients from 107 institutions between May 2013 and July 2015. The patients were randomized 2:1 to receive rocapuldencel-T plus standard therapy (primarily sunitinib) or standard therapy alone. The primary endpoint of the 
study is overall survival. In February 2017, it was announced that an Independent Data Monitoring Committee had recommended halting follow up based on a futility analysis at a time when $75 \%$ of the projected 290 deaths had occurred. ${ }^{36}$ Final analysis is projected in 2018.

The obvious weakness of our study was that it could not be completed as planned due to poor enrollment; therefore, the results are for only a small cohort of heterogenous patients. A major objective of this effort was to treat as many patients as possible for whom a tumor cell line had been generated. Therefore, it was anticipated that this patient cohort would be heterogenous in terms of extent of disease at the time of treatment because of variation in clinical courses from the time a sample of metastatic tumor was obtained to the time of actual treatment. Also, other than the tumor DTH skin testing, there was no evaluation of immune responses based on changes in lymphocytes or cytokines. As noted earlier, successful commercial application of this approach would necessitate a higher and faster rate of success in establishing autologous tumor cell lines.

\section{Hypothesis/Future Direction/Prospective and Prediction}

Cancer heterogeneity leads to the hypothesis that the optimal approach to personalized therapeutic vaccines is via autologous tumor antigens. Autologous DCs presenting such antigens appear to offer advantages over injections of irradiated autologous tumor cells themselves. Clinical results with autologous DCVs are encouraging but it is unclear what the optimal DC product is, and it is unclear whether strategies that enrich for tumor initiating cells as the source of antigen are superior to whole tumor lysate or mRNA as sources of autologous tumor antigens. Additional investigation and clinical trials are warranted.

\section{Conclusions}

In this study, there was evidence of clinical activity for a patientspecific DCV on the basis of two delayed complete remissions, and an observed 5-year survival rate of $33 \%$. This was achieved with minimal toxicity. Larger trials of DCs with autologous tumor antigens derived from self-renewing tumor cells, rather than bulk tumor, may be warranted, especially as an adjunct to other therapies, but the autologous tumor cell lines must be produced more rapidly and reliably for this to be practical.

\section{Acknowledgments}

We wish to acknowledge Linda D. Beutel, BS, Patric Schiltz, Ph.D., and Senthimil Selvan, Ph.D. for their manufacturing of products in the Hoag Cancer Center Cell Biology, and Kelly Austin, R.N. for performing leukapheresis procedures. We thank Kanoe Allen, R.N. for patient coordination, Cristina deLeon, B.S.N., CCRA, and Cheryl Mayorga L.V.N. for data collection, Khosrow Mahdavi, M.D. for referring two of the treated patients, and Candace Hsieh, Ph.D. for review of the manuscript.

\section{Conflict of interest}

RO Dillman is currently an employee of AIVITA Biomedical, Inc.
C Depriest has no conflicts of interest.

\section{Author contributions}

Dr. Dillman designed the clinical trial, directed the laboratory that manufactured the investigational products, analyzed the data, and wrote the manuscript. Ms. Depriest coordinated the clinical trial including product administration, monitoring of patients, and collection of data, and she reviewed the manuscript.

\section{References}

[1] Edge SE, Byrd DR, Compton CC, Fritz AG, Greene FL, Trotti A. Kidney in AJCC Cancer Staging Handbook 7th edition, New York, Springer, 2010, pp. 547-567.

[2] Medical Research Council Renal Cancer Collaborators. Interferonalpha and survival in metastatic renal carcinoma: early results of a randomised controlled trial. Lancet 1999;353(9146):14-17. doi:10.1016/S0140-6736(98)03544-2.

[3] Fyfe G, Fisher RI, Rosenberg SA, Sznol M, Parkinson Dr, Louie AC. Results of treatment of 255 patients with metastatic renal cell carcinoma who received high-dose recombinant interleukin-2 therapy. J Clin Oncol 1995;13(3):688-696. doi:10.1200/jco.1995.13.3.688.

[4] Lindsey KR, Rosenberg SA, Sherry RM. Impact of the number of treatment courses on the clinical response of patients who receive high-dose bolus interfleukin-2. J Clin Oncol 2000;18(9):1954-1959. doi:10.1200/JCO.2000.18.9.1954.

[5] McDermott DF, Regan MM, Clark JI, Flaherty LE, Weiss GR, Logan $\mathrm{TF}$, et al. Randomized phase III trial of high-dose interleukin-2 versus subcutaneous interleukin-2 and interferon in patients with metastatic renal cell carcinoma. J Clin Oncol 2005;23(1):133-141. doi:10.1200/jco.2005.03.206.

[6] Chow S, Galvis V, Pillai M, Leach R, Keene E, Spencer-Shaw A, et al. High-dose interleukin2 - a 10-year single-site experience in the treatment of metastatic renal cell carcinoma: careful selection patients gives an excellent outcome. J Immunother Cancer 2016;4:67. doi:10.1186/s40425-016-0174-5.

[7] NCCN Clinical Practice Guidelines in Oncology (NCCN Guidelines ${ }^{\circledR}$ ): Kidney Cancer. Version 3.2018; Available from: https://www.nccn. org/professionals/physician_gls/pdf/kidney.pdf, accessed February 06, 2018.

[8] Motzer RJ, Hutson TE, Tomczak P, Michaelson MD, Bukowski RM, Rixe $\mathrm{O}$, et al. Sunitinib versus interferon alfa in metastatic renal-cell cancer. N Engl J Med 2007;356(2):115-124. doi:10.1056/NEJMoa065044.

[9] Hudes G, Carducci M, Tomczak P, Dutcher J, Figlin R, Kapoor A, et al. Temsirolimus, interferon alfa, or both for advanced renal-cell carcinoma. N Engl J Med 2007;356(22):2272-2281. doi:10.1056/NEJMoa066838.

[10] Dillman RO. Cancer registry data for Hoag Hospital: 1998-2003 vs 2004-2009. Unpublished observations.

[11] Siegel RL, Miller KD, Jemal A. Cancer statistics, 2017. CA Cancer J Clin 2017;67(1):7-30. doi:10.3322/caac.21387.

[12] Motzer RJ, Escudier B, McDermott DF, George S, Hammers JH, Srinivas $\mathrm{S}$, et al. Nivolumab versus everolimus in advanced renal-cell carcinoma. N Engl J Med 2015;373(19):1803-1813. doi:10.1056/NEJMoa1510665.

[13] Motzer RJ, Tannir NM, McDermott DF, Arén Frontera O, Melichar B, Choueiri TK, et al. Nivolumab plus ipilimumab versus sunitinib in advanced renal-cell carcinoma. N Engl J Med 2018;378(15):1277-1290. doi:10.1056/NEJMoa1712126.

[14] Dillman RO. Is there a role for therapeutic cancer vaccines in the age of checkpoint inhibitors? Hum Vaccin Immunother 2017;13(3):528532. doi:10.1080/21645515.2016.1244149.

[15] Dillman RO, Cornforth AN, Nistor G. Cancer stem cell antigen-based vaccines: the preferred strategy for active specific immunotherapy of metastatic melanoma? Expert Opin Biol Ther 2013;13(5):643-656. d oi:10.1517/14712598.2013.759556. 
J Explor Res Pharmacol

[16] Therasse P, Arbuck SG, Eisenhauer EA, Wanders J, Kaplan RS, Rubinstein $L$, et al. New guidelines to evaluate the response to treatment in solid tumors. European Organization for Research and Treatment of Cancer, National Cancer Institute of the United States, National Cancer Institute of Canada. J Natl Cancer Inst 2000;92(3):205-216. doi:10.1093/jnci/92.3.205.

[17] Dillman RO, Nayak SK, Beutel L. Establishing in vitro cultures of autologous tumor cells for use in active specific immunotherapy. J Immunother Emphasis Tumor Immunol 1993;14(1):65-69.

[18] Dillman RO, Beutel LD, Cornforth, Nayak SK. Short-term tumor cell lines from renal cell carcinoma for use as autologous tumor cell vaccines in the treatment of kidney cancer. Cancer Biother Radiopharm 2000;15(2):161-168. doi:10.1089/cbr.2000.15.161.

[19] Nayak SK, Dillman RO. Establishment of multiple tumor cell lines from a patients with melanoma: a simple method to control fibroblast growth. Clin Biotechnol 1991;3:237-242.

[20] Dillman RO, Selvan SR, Schiltz PM, McClay EF, Barth NM, Depriest C, et al. Phase II trial of dendritic cells loaded with antigens from selfrenewing, proliferating autologous tumor cells as patient-specific anti-tumor vaccines in patients with metastatic melanoma: final Report. Cancer Biother Radiopharm 2009;24(3):311-319. doi:10.1089/ cbr.2008.0599.

[21] Cornforth AN, Fowler AW, Carbonell DJ, Fan E, Dillman RO. Characterization of interferon- $\gamma$-treated melanoma tumor cells for use in dendritic cell-based immunotherapy. Cancer Biother Radiopharm 2011;26(3):345-351. doi:10.1089/cbr.2011.0959.

[22] Dillman RO, Cornforth AN, Depriest C, McClay EF, Amatruda TT, de Leon $\mathrm{C}$, et al. Tumor stem cell antigens as consolidative active specific immunotherapy: a randomized phase II trial of dendritic cells versus tumor cells in patients with metastatic melanoma. J Immunother 2012;35(8):641-649. doi:10.1097/CJI.0b013e31826f79c8.

[23] Osband ME, Lavin PT, Babayan RK, Graham S, Lamm DL, Parker $B$, et al. Effect of autolymphocyte therapy on survival and quality of life in patients with metastatic renal-cell carcinoma. Lancet 1990;335(8696):994-998. doi:10.1016/0140-6736(90)91064-H.

[24] Berntsen A, Geersen, Svane IM. Therapeutic dendritic cell vaccination of patients with renal cell carcinoma. Euro Urol 2006;50(1):3443. doi:10.1016/j.eururo.2006.03.061.

[25] Draube A, Klein-Gonalez N, Mattheus S, Brillant C, Hellmich M, Engert $A$, et al. Dendritic cell based tumor vaccination in prostate and renal cell cancer: a systematic review and meta-analysis. PLoS
Dillman RO. et al: Dendritic cell vaccine for renal cell cancer

One 2011;6(4):e18801. doi:10.1371/journal.pone.0018801.

[26] Dillman RO, Cornforth AN, Nestor GI. Dendritic cell vaccines for melanoma: past, present and future. Melanoma Manage 2016;3(4):267283. doi:10.2217/mmt-2016-0014.

[27] Dillman R, Barth N, VanderMolen L, Mahdavi K, Beutel L, de Leon C, et al. Autologous tumor cell line-derived vaccine for patient-specific treatment of advanced renal cell carcinoma. Cancer Biother Radiopharm 2004;19(5):570-578. doi:10.1089/cbr.2004.19.570.

[28] Dillman RO, Cornforth AC, Nistor GI, McClay EF, Amatruda TT, Depriest C. Randomized phase II trial of autologous dendritic cell vaccines versus autologous tumor cell vaccines in metastatic melanoma: 5-year follow up and additional analyses. J Immunother Cancer 2018;6(1):19. doi:10.1186/s40425-018-0330-1.

[29] Schendel DJ. Dendritic cell vaccine strategies for renal cell carcinoma. Expert Opin Biol Ther 2007;7(2):221-232. doi:10.1517/ 14712598.7.2.221.

[30] Yoshimura K, Uemura H. Role of vaccine therapy for renal cell carcinoma in the era of targeted therapy. Int J Urol 2013;20(8):744-755. doi:10.1111/iju.12147.

[31] Kirner A, Mayer-Mokler A, Reinhardt C. IMA901: a multi-peptide cancer vaccine for treatment of renal cell cancer. Hum Vaccin Immunother 2014;10(11):3179-3189. doi:10.4161/21645515.2014.983857.

[32] Soleimani A, Berntsen A, Svane IM, Pedersen AE. Immune responses in patients with metastatic renal cell carcnoma treated with dendritic cells pulsed with tumor lysate. Scand J Immunol 2009;70(5):481489. doi:10.1111/j.1365-3083.2009.02322.x.

[33] Kugler A, Stuhler G, Walden P, Zöller G, Zobywalski A, Brossart P, et al. Regression of human metastatic renal cell carcinoma after vaccination with tumor cell-dendritic cell hybrids. Nat Med 2000;6(3):332-336.

[34] Gitlitz BJ, Belldegrun AS, Zisman A, Chao DH, Pantuck AJ, Hinkel $A$, et al. A pilot trial of tumor lysate-loaded dendritic cells for the treatment of metastatic renal cell carcinoma. J Immunother 2003;26(5):412-419.

[35] Su Z, Dannull J, Heiser A, Yancey D, Pruitt S, Madden J, et al. Immunological and clinical responses in metastatic renal cancer patients vaccinated with tumor RNA-transfected dendritic cells. Cancer Res 2003;63(9):2127-2133.

[36] Argos press release. Independent data monitoring committee recommends discontinuation of the ADAPT phase 3 clinical trial of rocapuldencel-t in metastatic renal cell carcinoma for futility following its planned interim data review. 22 February 2017. 